\title{
A Man With Abdominal Pain and Acute Renal Failure
}

Faisal Shaikh MD, Marina Serper, $M D$

\section{Case Presentation}

A 33 year-old African American male with a past medical history significant for HIV on HAART therapy presented to the emergency department (ED) with acute onset of right upper quadrant abdominal pain and mild shortness of breath. The abdominal pain was not associated with any fevers, chills, nausea, vomiting or diarrhea. The patient denied any chest pain, palpitations, lightheadedness or syncopal episodes. He did report some dysphagia with solids more than liquids over the last 2 months as well as decreased oral intake. His review of systems was otherwise significant for weight loss which he was unable to quantify.

He denied any medication allergies. His medications on admission included trimethoprim/sulfmethaxazole, azithromycin, fluconazole, lopinavir/ritonavir, and emtricitabine/ tenofovir. His most recent CD4 count was 114 cells $/ \mathrm{mm}^{3}$ and he had no other known past medical history.

On initial physical exam, the patient was found to be tachycardic at $115 \mathrm{bpm}$, hypotensive with a blood pressure of $80 / 40 \mathrm{~mm}$ $\mathrm{Hg}$, and tachypneic exhibiting $32 \mathrm{resps} / \mathrm{min}$. The head and neck exam were significant for oral thrush. Other than resting tachycardia, the cardiovascular exam was unremarkable. The pulmonary exam was notable for tachypnea, but the lungs were clear to auscultation and there was no observed accessory muscle use. The abdominal exam was significant for tenderness to palpation in the right upper quadrant without rebound or guarding. The patient was alert and oriented and had no focal neurological deficits.

Admission labs were notable for a white blood cell count of 19.3 cells $/ \mu \mathrm{L}$ with $22 \%$ bands, creatinine $1.8 \mathrm{mg} / \mathrm{dL}$ (no baseline available), total bilirubin $1.8 \mathrm{mg} / \mathrm{dL}$, direct bilirubin $1.2 \mathrm{mg} / \mathrm{dL}$ with an alkaline phosphatase of 1233 IU/L. AST and ALT were $90 \mathrm{U} / \mathrm{L}$ and $64 \mathrm{U} / \mathrm{L}$, respectively. The patient was also noted to have a lactate of $86.7 \mathrm{mEq} / \mathrm{L}$.

\section{Hospital Course}

The patient responded to 3L normal saline fluid resuscitation to $110 / 60 \mathrm{~mm} \mathrm{Hg}$. Broad spectrum antibiotics (vancomycin and pipercillin/tazobactam) were begun and the patient was admitted to a telemetry floor. Initial studies included an abdominal ultrasound which showed 2 hypoechoic masses in the left lobe of the liver without intra- or extrahepatic ductal dilatation noted. A non-contrast CT of the abdomen and pelvis demonstrated innumerable lymph nodes scattered diffusely throughout the mesentery and retroperitoneum measuring up to $1.5 \mathrm{~cm}$, likely related to the history of HIV infection. A renal ultrasound showed normal sized kidneys, and no hydronephrosis. The nephrology service was consulted and the working diagnosis at the time was renal failure secondary to tenofivir.
The tenofivir was discontinued and his abdominal pain workup was continued.

The hospital course was notable for diarrhea, progressive lethargy, fevers up to $104^{\circ} \mathrm{F}$, worsening abdominal pain, continued rise in serum creatinine to $5.1 \mathrm{mg} / \mathrm{dL}$, and decreased urine output to 200 cc's over 24 hours. On hospital day 3, the patient was transferred to the intensive care unit after an arterial blood gas demonstrated a profound metabolic acidosis characterized by a $\mathrm{pH}$ 7.15. A repeat non- contrast CT of abdomen and pelvis now revealed diffuse, marked small bowel and large bowel wall thickening with two focal segments suggestive of small bowel intussusceptions without bowel obstruction.

The patient's abnormal though non-specific intra-abdominal findings in the setting of worsening acidosis and elevated lactate levels prompted an exploratory laparotomy. Seven areas of easily reducible intussusceptions were discovered. In fact, innumerable mesenteric lymph nodes were found, believed to act as lead points for the pathogenesis of these intussusceptions. Fortunately, the bowel was still viable avoiding the need for resection.

Post-op, the patient continued to be oliguric with severe electrolyte imbalances (Urate 27.5, K $6.2 \mathrm{mEq} / \mathrm{L}, \mathrm{Ca}^{2+} 3.0 \mathrm{mEq} /$ $\left.\mathrm{L}, \mathrm{PO}_{4}^{3-} 17.4 \mathrm{mEq} / \mathrm{L}, \mathrm{LDH} 1154 \mathrm{mEq} / \mathrm{L}\right)$. Spontaneous tumor lysis syndrome was diagnosed and the patient was begun on emergent hemodialysis. Intra-operatively obtained mesenteric lymph nodes demonstrated atypical lymphoid cells with a high mitotic rate that focally demonstrate a "starry-sky" pattern consistent with a diagnosis of Burkitt lymphoma. Due to the patient's poor functional \& physiologic status, it was decided that he was a poor candidate for chemotherapy. The patient elected no other therapies and elected for hospice care.

\section{Discussion}

Burkitt lymphoma is a rare disorder comprising less than $1 \%$ of non Hodgkin B cell lymphomas. First described by Dr. Dennis Burkitt in Uganda in 1958, it is an aggressive lymphoma frequently presenting in extranodal sites, or as a leukemia, characterized by monomorphic medium sized cells with a basophilic cytoplasm and very high proliferation rate. It is classified by three major types: endemic, sporadic, and immunodeficient. ${ }^{1,2}$

The endemic form is most common in Africa and New Guinea, where is it the most common childhood neoplasm. The endemic form typically affects facial bones in children (mean age 4-7) and is associated with EBV more than $95 \%$ of the time. The sporadic form occurs most commonly in developed countries and a mean age of onset of 30 . The sporadic form arises from lymphoid tissue in the gut and respiratory tract with most cases being extranodal and jaw involvement less than $30 \%$ of the time. The immunodeficient form is associated with HIV infection and 
most commonly affects the lymph nodes and bone marrow. Paradoxically, these patients typically have a high CD4 count with few opportunistic infections.

The clinical presentation is varied and nonspecific, but frequently involves the abdomen (i.e. pain, ascites). Patients may also present with classic B symptoms of fevers, night sweats, and weight loss. The diagnosis is confirmed with lymph node biopsy, bone marrow biopsy, and lumbar puncture (the last two necessary for deciding on treatment options).

The biology of the disease involved chromosomal translocation and dysregulation of the $c-m y c$ gene, which controls cellular proliferation. ${ }^{1}$ Tumor cells express a high level of the ki 67 protein indicating high levels of mitotic activity. On pathology, sheets of $B$ cells are seen with frequent mitotic figures classically giving the tumor cells a "starry sky" appearance. It is important to differentiate between Burkitt and diffuse large B cell lymphoma (which can sometimes have a similar pathologic appearance) because the former is a more aggressive type of lymphoma requiring intrathecal chemotherapy. Recently, molecular gene signatures have been used to aid in this differentiation.

Typically, the Ann Arbor system is used in staging lymphomas; however, Burkitt lymphoma does not lend itself well to the staging system due to its extremely aggressive course. Due to the nature of the tumor, chemotherapy needs to be initiated as soon as possible, preferably within 48 hours of diagnosis. A standard regimen includes cyclophosphamide, vincristine, doxorubicin, and high dose methotrexate and intrathecal therapy with alternating ifosfamide, etoposide, and high dose cytarabine. In one study, this regimen showed a $92 \%$ two year event free survival in children and adults. ${ }^{3}$ The significant side effects experienced were neurotoxicity, bone marrow suppression, severe mucositis, and tumor lysis syndrome. Another study showed that rituximab, a CD20 monoclonal antibody, further increased two year event free survival (80-88\%) when added to the standard regimen. Studies are now under way with other monoclonal antibodies and other biologic agents. There is no clear role for bone marrow transplantation in the treatment of the disease.

Once entertained, the diagnosis of Burkitt lymphoma should be promptly confirmed with prompt initiation of chemotherapy to combat the aggressive nature of this specific type of lymphoma. New monocloncal antibody agents currently offer new survival benefits not previously seen before.

\section{References}

1. Molecular Diagnosis of Burkitt's Lymphoma. Dave SS, Fu K, Wright GW, Lam LT, Kluin P, Boerma EJ, Greiner TC, Weisenburger DD, N Engl J Med 2006; 354:2431.

2. Biology ad treatment of Burkitt's lymphoma. Yustein J, Dang Chi. Curren Opin Hematol 2007; 14:375-381.

3. Magrath et al. Adults and children with small noncleaved-cell lymphoma have a similar excellent outcome when treated with the same chemotherapy regimen. J Clin Oncol 1996;14:925-934 